\title{
Approvisionnement en eau dans la commune des Parcelles Assainies de Dakar (Sénégal): perception de la qualité et pratiques des populations
}

\author{
Cheikh Diop, PhD \\ Aminata Toure, PhD \\ Fatoumata Bah, PhD \\ Absa Lam, PhD \\ Mathilde Cabral, PhD \\ Seynabou Fedior, PharmD \\ Mamadou Fall, PhD
}

Université Cheikh Anta DIOP de Dakar, Faculté de Médecine, Pharmacie et Odontologie, Laboratoire de Toxicologie et Hydrologie, Dakar Fann, Sénégal

Doi:10.19044/esj.2021.v17n7p256

Submitted: 27 January 2021

Accepted: 27 February 2021

Published: 28 February 2021
Copyright 2021 Author(s)

Under Creative Commons BY-NC-ND

4.0 OPEN ACCESS

Cite As:

Diop C., Toure A., Bah F., Lam A., Cabral M., Fedior S. \& Fall M. (2021).

Approvisionnement en eau dans la commune des Parcelles Assainies de Dakar (Sénégal): perception de la qualité et pratiques des populations. European Scientific Journal, ESJ, 17(7), 256. https://doi.org/10.19044/esj.2021.v17n7p256

\section{Résumé}

L'eau est indispensable pour satisfaire les besoins domestiques et alimentaires, mais sa disponibilité permanente est une préoccupation réelle en milieu urbain notamment dans les quartiers défavorisés des pays en développement. Le manque d'eau et la qualité de celle-ci exposent les populations particulièrement les enfants aux maladies hydriques. L'objectif de ce travail est d'identifier les différentes sources d'approvisionnement en eau et d'évaluer la perception de la qualité de l'eau et son impact sur la santé par les populations de la commune des Parcelles Assainies de Dakar. La méthodologie adoptée dans cette étude est basée sur une enquête qui a consisté à administrer aux ménages un questionnaire pré-validé. La méthode des quotas a permis de sélectionner 400 ménages répartis dans les différents quartiers de la commune. Les résultats indiquent que $82,3 \%$ de la population de la commune s'approvisionnent avec l'eau du robinet et 70,5\% utilisent des 
pompes manuelles en cas de coupure d'eau. L'eau du robinet sert essentiellement de boisson (73\%) et celle des pompes manuelles pour la douche, le linge et les travaux ménagers. La majorité de la population (56\%) trouve que la qualité de l'eau est moyennement bonne, $24 \%$ indiquent qu'elle est bonne et $20 \%$ considèrent qu'elle est mauvaise. L'odeur est le paramètre organoleptique le plus utilisé par la population $(68 \%)$ pour apprécier la qualité de l'eau suivi du goût $(63 \%)$ et de la couleur (62\%). Cette situation justifie le traitement de l'eau à domicile avant usage avec l'eau de javel $(66,7 \%)$, de comprimés désinfectant Aquatabs ${ }^{\circledR}(32,2 \%)$, la filtration et la décantation $(21,9 \%)$. Par ailleurs, $75 \%$ des enquêtés trouvent que la fréquence des maladies diarrhéiques chez les enfants est liée à la qualité de l'eau. L'analyse des résultats montre une diversité des sources d'approvisionnement liée au manque de moyens économiques associés aux fréquentes coupures d'eau du robinet, une mauvaise perception de la qualité de l'eau et une fréquence élevée de maladies liée à l'eau chez les enfants.

Mots clés : Eau, approvisionnement, qualité, maladies hydriques, perception, Dakar

\title{
Water Supply in the Commune of Parcelles Assainies de Dakar (Senegal): Perception of the Quality and Practice of The Populations
}

\author{
Cheikh Diop, PhD \\ Aminata Toure, PhD \\ Fatoumata Bah, PhD \\ Absa Lam, PhD \\ Mathilde Cabral, PhD \\ Seynabou Fedior, PharmD \\ Mamadou Fall, PhD
}

Université Cheikh Anta DIOP de Dakar, Faculté de Médecine, Pharmacie et Odontologie, Laboratoire de Toxicologie et Hydrologie, Dakar Fann, Sénégal

\begin{abstract}
Water is essential to meet domestic and food needs, but its permanent availability is a real concern in urban areas, particularly in disadvantaged neighborhoods in developing countries. The lack of water and its quality exposes populations, particularly children, to water-borne diseases. The objective of this work is to identify the different sources of water supply and to evaluate the perception of water quality and its impact on health by the
\end{abstract}


populations of the commune of Parcelles Assainies de Dakar. The methodology adopted in this study is based on a survey that consisted of administering a pre-validated questionnaire to households. The quota method made it possible to select 400 households distributed in the different neighborhoods of the commune. The results indicate that $82.3 \%$ of the commune's population is supplied with tap water and $70.5 \%$ use hand pumps in the event of water cuts. Tap water is mainly used for drinking (73\%) and hand pumps for showering, laundry and housework. The majority of the population $(56 \%)$ finds the water quality to be moderately good, $24 \%$ indicate that it is good and $20 \%$ consider it to be poor. Odour is the organoleptic parameter most used by the population (68 \%) to assess water quality, followed by taste $(63 \%)$ and color $(62 \%)$. This situation justifies treating water at home before use with bleach $(66.7 \%)$, Aquatabs $®$ disinfectant tablets (32.2\%), filtration and decantation $(21.9 \%)$. In addition, $75 \%$ of respondents felt that the frequency of diarrheal diseases in children is related to water quality. Analysis of the results shows a diversity of sources of supply linked to the lack of economic means associated with frequent tap water cuts, poor perception of water quality and a high frequency of water-related illnesses among children.

Keywords: Water, supply, quality, waterborne diseases, perception, Dakar

\section{Introduction}

L'approvisionnement en eau potable est l'une des questions les plus stratégiques et les plus délicates de notre temps. L'eau est indispensable à la vie, la terre ou « planète bleue » est constituée à plus de $70 \%$ de terre immergée; mais cette eau est en majorité salée (eaux marines, eaux océaniques et eaux des lacs salés) et non potable. L'eau des cours d'eau (fleuves, lacs d'eau douce, rivières, ruisseaux etc.) ne représente que moins de $0,1 \%$ des réserves d'eau de la planète et est souvent polluée. L'eau majoritairement consommée par la grande partie des Hommes provient pour l'essentiel des nappes aquifères ou souterraines et pose des problèmes d'accessibilité et d'exploitation (Brooks, 2002). De ces faits, l'approvisionnement en eau potable est devenu un des enjeux majeurs pour les sociétés contemporaines surtout dans les zones urbaines où près de la moitié de la population mondiale vit aujourd'hui. En 2017, on estimait que 785 millions d'êtres humains n'avaient pas accès à l'eau potable (UNICEF et OMS, 2019). Aussi, la population mondiale qui devrait passer de six milliards d'individus en l'an 2000 à huit milliards en 2025 va entraîner une baisse d'un tiers de la quantité moyenne d'eau douce disponible qui est de 6600 $\mathrm{m}^{3} / \mathrm{habitant/an}$. De plus, la consommation d'eau est inégalitaire, elle est en moyenne de 20 litres par personne et par jour dans les pays en développement 
contre 400 à 500 litres par personne dans les pays industrialisés (Roch, 2008). Face à la croissance démographique accélérée des pays en développement en général et en particulier en milieu urbain, l'amélioration de l'approvisionnement en eau potable des populations apparait donc indispensable. En Afrique, la population qui n'a pas accès à une source d'eau améliorée a augmenté, passant 319 millions en 2015 à 400 millions en 2017 (UNICEF et OMS, 2019). L'Afrique subsaharienne, réputée par ces espaces désertiques, est la partie de l'Afrique la plus touchée par le problème d'approvisionnement en eau potable avec la raréfaction de cette ressource. Elle connaît ces dernières décennies une baisse notable de la pluviométrie, combinée à une démographie galopante et une urbanisation rapide et non maîtrisée. En 2017, seul 61 \% de la population en Afrique subsaharienne avait accès à l'eau potable (UNICEF et OMS, 2019). Au Sénégal, malgré la modernisation et l'amélioration de la distribution de l'eau afin de rendre celleci accessible à toute la population, certains ménages s'approvisionnent avec d'autres sources d'eau dont la qualité est douteuse. Cette pratique s'observe aussi bien en milieu rural qu'urbain. Ainsi, si pour certaines populations Dakaroises, le problème de l'accès à l'eau potable ne se pose que sommairement ; pour d'autres (les habitants des Parcelles Assainies, par exemple), les difficultés d'approvisionnement en eau potable sont profondes et récurrentes.

La commune des Parcelles Assainies s'alimente en eau potable pour l'essentiel grâce au réseau de distribution public. Selon l'Enquête Démographique et de Santé (EDS), plus de $82 \%$ des ménages consomment de l'eau provenant d'une source améliorée et dans la majorité des cas, l'eau utilisée provient d'un robinet; $45 \%$ des ménages disposent de toilettes améliorées non partagées contre $32 \%$ de ménages qui utilisent des toilettes non améliorées et dans la majorité des cas, des latrines traditionnelles (ANSD, 2018). Par ailleurs, avec les coupures fréquentes de la fourniture d'eau, les populations puisent l'eau de la nappe phréatique souvent peu profonde et exposée à la pollution pour la boisson sans aucun traitement ou avec un traitement sommaire. Cete situation les expose à des problèmes de salubrité et de santé puisque l'inaccessibilité de l'eau potable et le défaut d'assainissement sont fortement associés à des pathologies d'origine hydrique comme la diarrhée surtout chez les enfants âgés de moins de cinq ans (Kaboré et al., 2017 ; He et al., 2018 ; Thiam et al., 2019).

Ainsi, ce travail a pour objectif d'identifier les différentes sources d'approvisionnement en eau des Parcelles Assainies et d'évaluer la perception de la qualité de l'eau et de son impact sur la santé des enfants. 


\section{Matériels et méthodes}

\subsection{Présentation de la zone d'étude}

Cette étude a été réalisée dans la commune des Parcelles Assainies qui est l'une des 19 communes d'arrondissement de la Ville de Dakar (Sénégal). Elle se situe au Nord-Est de la capitale à moins d'une dizaine de kilomètres du Centre-ville et fait partie de la banlieue périurbaine de Dakar (Figure 1). Sa superficie est de $4,07 \mathrm{~km}^{2}$ et elle compte un peu plus de 300000 habitants répartis sur 20 unités de voisinage ou quartiers, chacun composé d'au moins 550 concessions. La commune des Parcelles Assainies constitue aujourd'hui un espace très hétérogène du point de vue de sa composition ethnique et socioprofessionnelle et on y trouve toutes les couches de la population sénégalaise (ANSD, 2016).

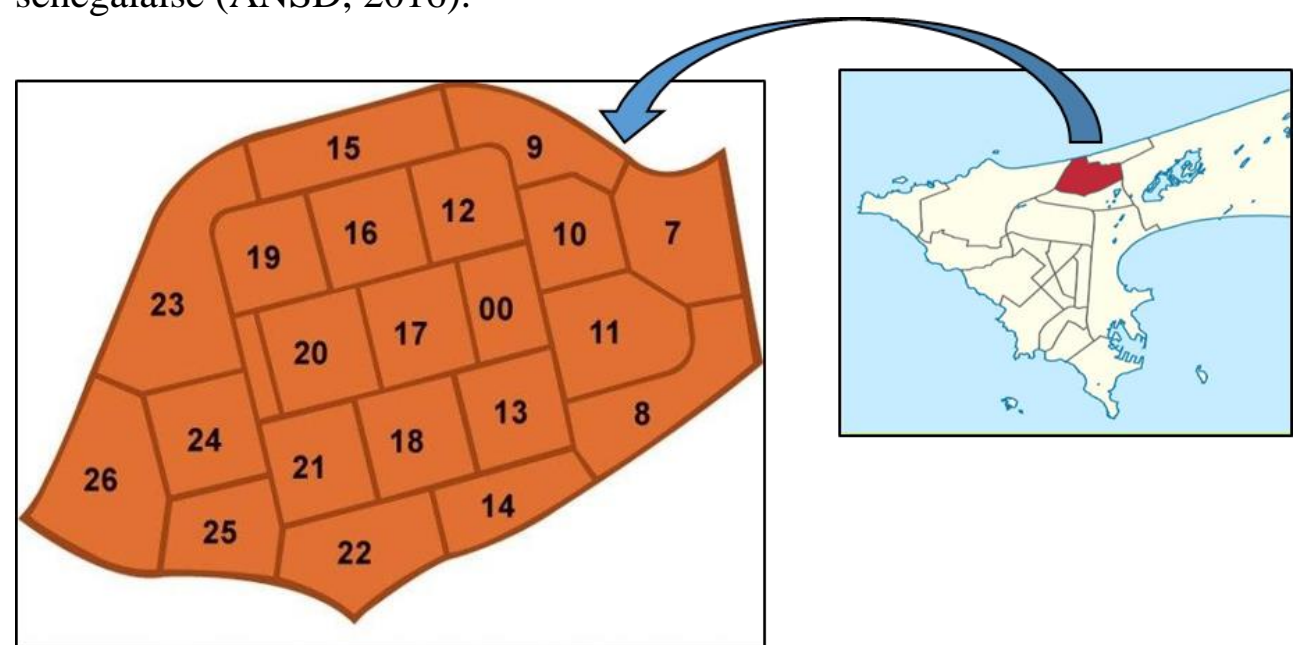

Figure 1 : Localisation de la zone d'étude

\subsection{Méthode d'étude}

\subsubsection{Période et type d'étude}

L'étude s'est déroulée dans les ménages de la commune des Parcelles Assainies du 15 février au 12 mars 2019. Il s'agit d'une étude transversale de type descriptif et prospectif.

\subsubsection{Population d'étude et critères de sélection}

Dans ce travail, les chefs de ménage de la commune constituent les principales cibles. Le ménage est défini dans cette étude comme un groupe de personnes qui vivent ensemble sous le même toit et mettent en commun tout ou une partie de leurs ressources pour subvenir à leur besoin essentiel, notamment le logement et la nourriture. Les questionnaires ont été administrés auprès de 400 chefs de ménage représentatifs de la population répartis entre les 21 quartiers de la commune. Cette représentativité de la taille de l'échantillon a été assurée par la méthode des quotas au regard du nombre de 
ménages par quartier sur la base des données de projection démographique de 2016 (ANSD, 2016). La sélection des ménages a été effectuée en respectant le pas de 5 ménages et le choix d'une personne par ménage. Pour faire partie de l'échantillon d'étude, il faut être un membre desdits ménages et résidant aux Parcelles Assainies depuis plus d'un an et capable de répondre aux questions. Toute personne jugée inéligible pour répondre aux questions et/ou résidant aux Parcelles Assainies depuis moins d'un an est exclue de l'étude.

\subsubsection{Collecte et traitement des données}

Une fiche de saisie des données sous forme de questionnaire est l'instrument de collecte utilisé dans cette étude. Elle a été élaborée de manière à permettre d'apporter des réponses adéquates aux différents questionnements. Une enquête pilote effectuée a permis d'identifier les difficultés liées à l'administration du questionnaire aux enquêtés et d'y apporter des corrections. Le recueil des données a été fait lors d'un entretien individuel dans un endroit discret permettant de garantir la confidentialité des informations fournies par l'enquêté. L'exploitation des données a été faite à l'aide des logiciels Excel version 2013 et SPSS 17.0 (SPSS, Paris, France) utilisés pour les données quantitatives, les tableaux et le traitement statistique.

\section{Résultats et discussion}

\subsection{Données sociodémographiques}

Selon l'Agence Nationale de la Statistique et de la Démographie (ANSD) en 2017, la population de Dakar était de 3529300 habitants dont 1 758957 hommes et 1770343 femmes. Donc notre étude faite sur un échantillon de 400 personnes constitué de $62 \%$ d'hommes et $38 \%$ de femmes représente $0,011 \%$ de la population de Dakar. Ces résultats sont similaires à ceux de la littérature qui montrent que 60 à $65 \%$ des ménages sont dirigés par des hommes (Barou, 2017 ; ANSD, 2016). 88 \% des chefs de ménage enquêtés étaient âgés de plus de 25 ans.

\subsection{Identification des sources d'eau}

La figure 2 montre les différentes sources d'eau que les populations de la commune exploitent pour les besoins domestiques. 


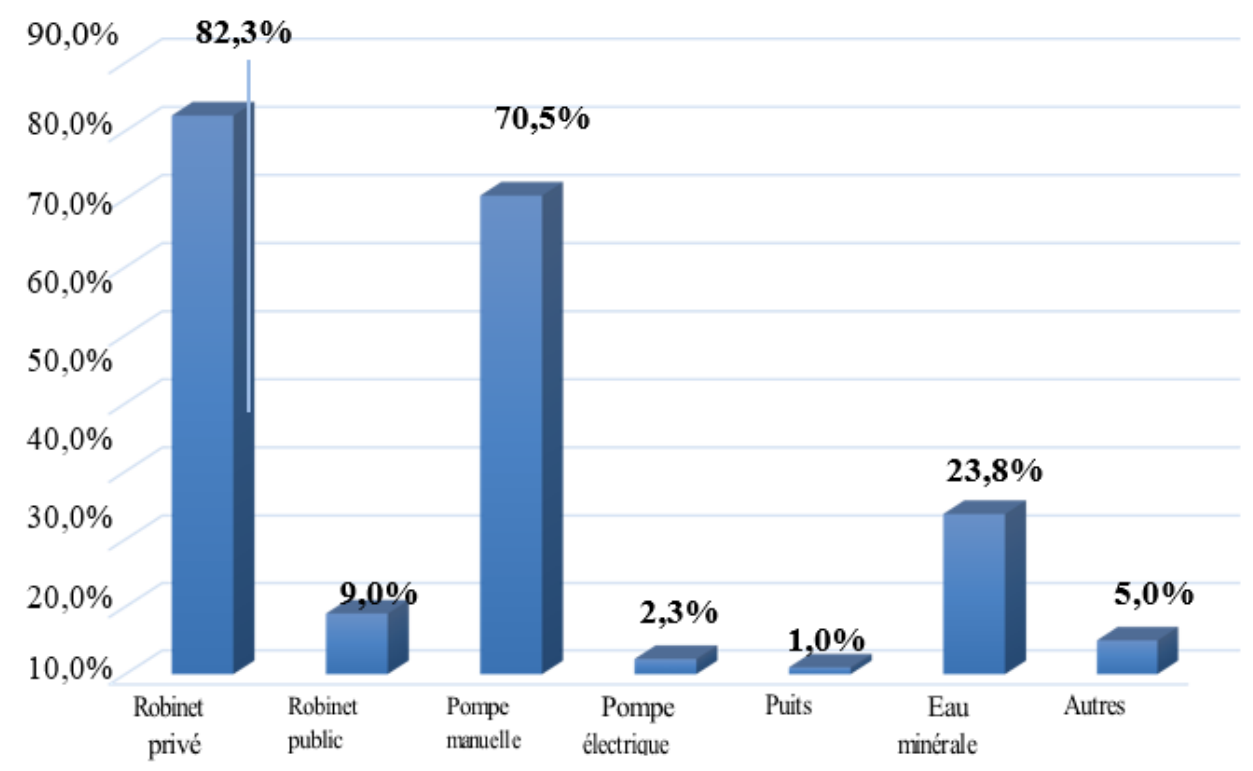

Figure 2 : Sources d'approvisionnement en eau des Parcelles Assainies

La presque totalité de la population de la commune des Parcelles Assainies s'approvisionne avec l'eau du robinet privé (82,3\%). L'utilisation des pompes manuelles est importante avec un taux de 70,5\%. La consommation de l'eau minérale représente $23,8 \%$ de la population étudiée alors que $9 \%$ utilisent les robinets publics. L'utilisation des pompes électriques devient de plus en plus courante $(2,3 \%)$ tandis que les puits sont presque abandonnés (1\%) (Figure 2). Cette situation est à l'opposé de celle de la commune de Fatick où deux modes d'approvisionnement en eau dominent: l'approvisionnement à travers le réseau public qui comprend les branchements privés et l'approvisionnement par les puits (Sène, 2012). Une étude réalisée à Brazzaville au Congo montre que les sources d'approvisionnement en eau sont diversifiées à cause des situations de pénurie et/ou de coupure d'eau répétée enregistrées par les ménages (Nkounkou et al., 2017). Contrairement à nos résultats, une étude menée à Yaoundé au Cameroun révèle que la principale source d'approvisionnement en eau des populations est le puits du fait de coût élevé de la connexion au réseau d'adduction (Tchouongsi et al., 2020)

\subsection{Types d'usage de l'eau selon la source}

S'agissant de l'utilisation des différentes sources d'eau, la figure 3 indique que la population d'étude boit principalement l'eau du robinet $(73 \%)$. La cuisine est assurée avec l'eau du robinet privé (71\%) et celle des pompes manuelles $(25 \%)$. Ces dernières sont plus utilisées pour la douche, le linge et 
les travaux domestiques (70 \% des ménages). Or, dans une commune comme celle de Fatick, l'eau des puits constitue la principale source d'alimentation en eau potable des ménages puisqu'utilisée par $99 \%$ de la population pour la boisson contre $1 \%$ qui utilise celle du robinet (Sène, 2012). Selon (SantsaNguefack et al., 2018), cette situation serait due non seulement à la couverture insuffisante du réseau de distribution public mais aussi aux faibles revenus de certains habitants. Des situations similaires d'usage de l'eau selon la source d'approvisionnement ont été décrites à Grand-Popo au Bénin, à Pointe-Noire au Congo, à Yaoundé et à Mbouda au Cameroun (Makoutode et al., 1999; Hanus, 2005 ; Nanfack et al., 2014).

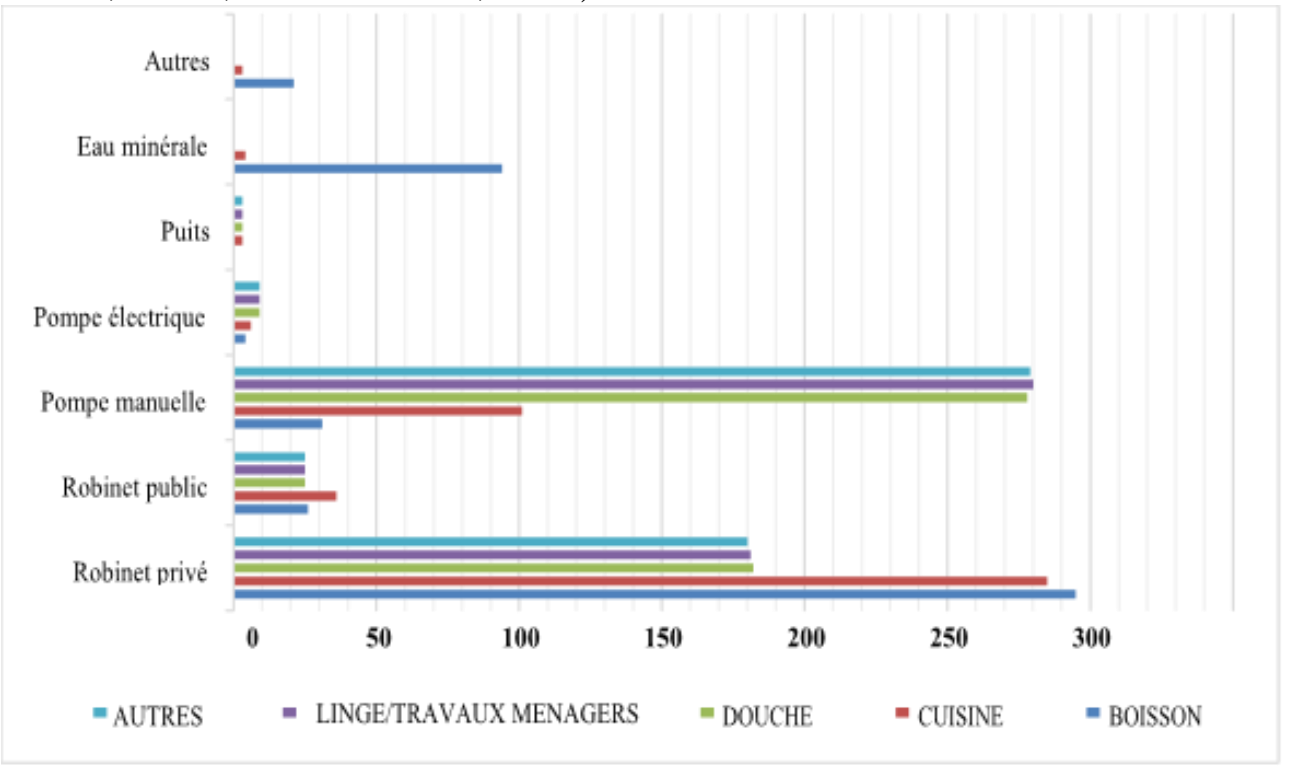

Figure 3 : Types d'usage de l'eau selon la source

\subsection{Raisons d'utilisation des différentes sources d'eau}

Pour notre étude, l'utilisation de chaque type de source d'eau répond à une des raisons répertoriées dans la figure 4 . 


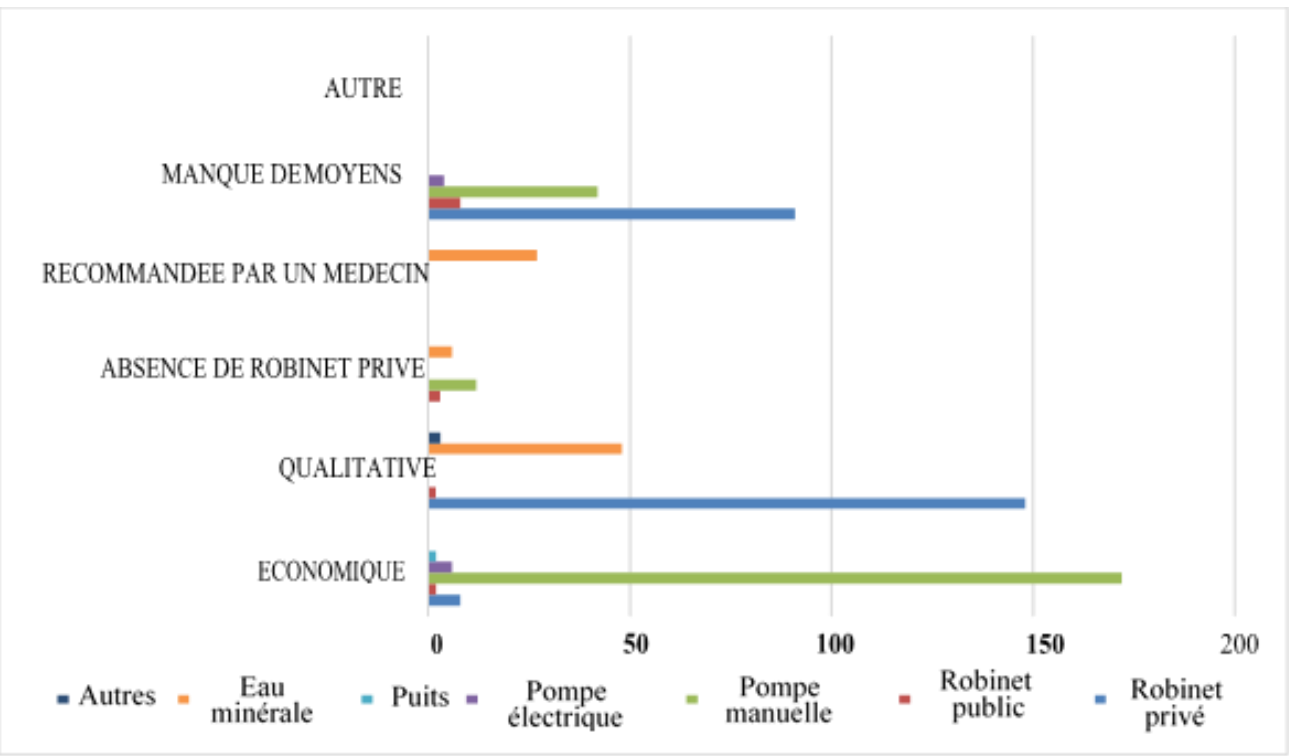

Figure 4 : Motifs d'usage de chaque source d'eau

Le manque de moyens et les problèmes économiques associés à la fréquence des coupures d'eau du robinet poussent la plupart des populations des Parcelles Assainies à faire usage des pompes manuelles. De telles observations ont été décrites en Côte d'ivoire (Santsa-Nguefack et al., 2018). Par ailleurs, une bonne partie de la population considère que l'eau du robinet est de meilleure qualité que celle des pompes mais elle n'est pas toujours disponible. Avec plus de moyens, la majorité de la population aurait abandonné cette eau du robinet au profit de celle minérale qui est généralement utilisée lorsqu'elle est recommandée par un médecin. En effet, ces eaux doivent avoir des propriétés favorables à la santé (Pepoff, 2010 ; Dupont et al., 2014). Dans la commune de Fatick, l'eau des puits est privilégiée à celle du robinet à cause du non-fonctionnement de $85 \%$ des bornes fontaines établies dans la commune et surtout de sa mauvaise qualité organoleptique (Sène, 2012).

\subsection{Perception de la qualité des eaux}

L'évaluation de la qualité de l'eau potable d'un réseau de distribution est souvent faite en tenant compte des normes physico-chimiques et microbiologiques édictées par les réglementations nationales. Etant la plus usitée dans la commune, la qualité de l'eau de robinet a été appréciée par la population d'étude. $56 \%$ trouve que la qualité de l'eau du robinet est moyennement bonne, $20 \%$ la considère de mauvaise qualité, par contre, 24 $\%$ de la population indique qu'elle est de bonne qualité (Figure 5). 


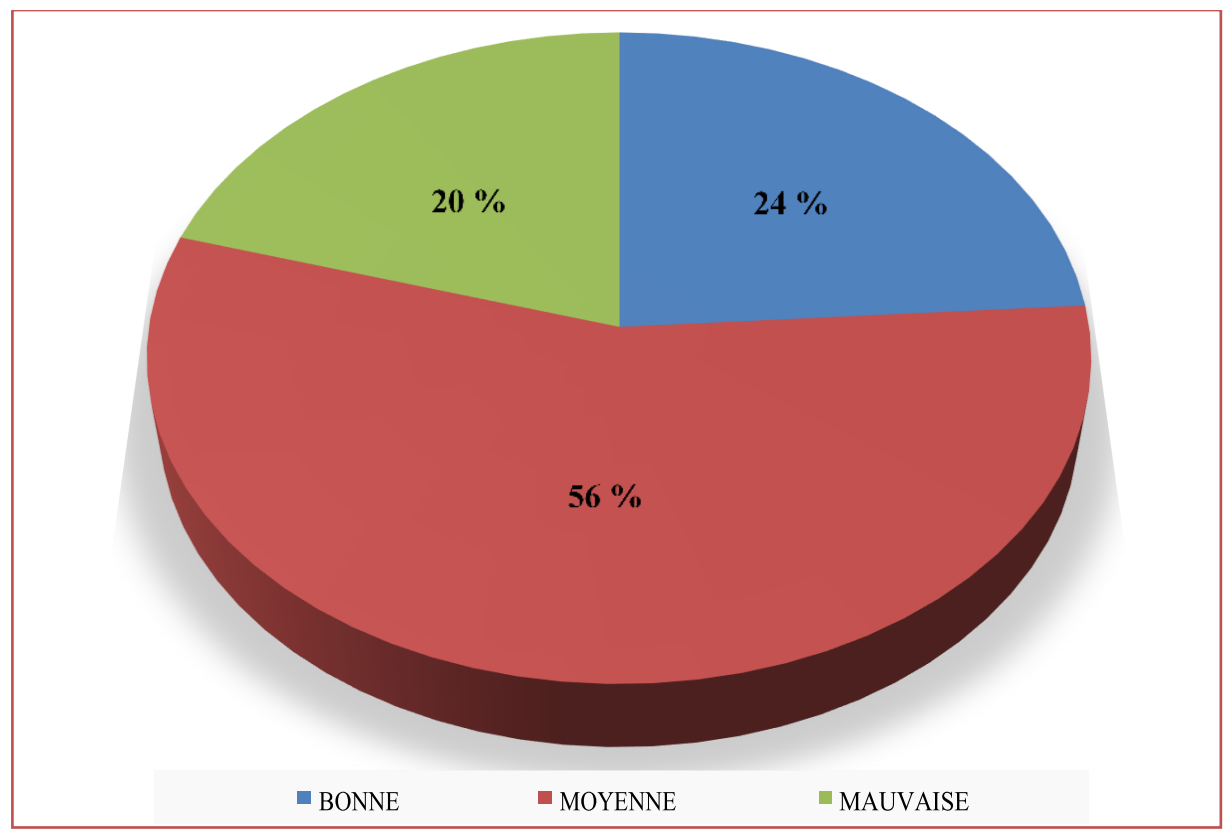

Figure 5 : Perception de la qualité de l'eau par la population

Ce résultat montre que malgré les efforts de la société de distribution de l'eau pour se conformer à la réglementation nationale, les consommateurs renoncent fréquemment à consommer l'eau du robinet du fait de la qualité organoleptique (goût, odeur) de l'eau distribuée (Proulx et al., 2010). Des études menées dans les communes de Cocody et Yopougon en Côte d'ivoire montrent que $90 \%$ des personnes enquêtées ne sont pas satisfaites de la limpidité de l'eau de boisson distribuée (Ballet et al., 2018).

Toutefois, la qualité de l'eau semble être meilleure dans la commune des Parcelles Assainies que dans celle de Fatick où la population affirme ne pas boire l'eau du robinet à cause de sa qualité. En effet, dans la commune de Fatick, les teneurs élevées de fluorure $(1,8 \mathrm{mg} / \mathrm{L})$ dans les eaux distribuées au robinet sont à l'origine de maladies (fluorose) chez les populations consommant cette eau ; ce qui situe la commune dans la zone de fluorose endémique (Caritas Sénégal, 2009).

\subsection{Paramètres d'appréciation de la qualité des eaux}

L'appréciation de la qualité de l'eau a été soumise à certains critères recensés dans la figure 6 . 


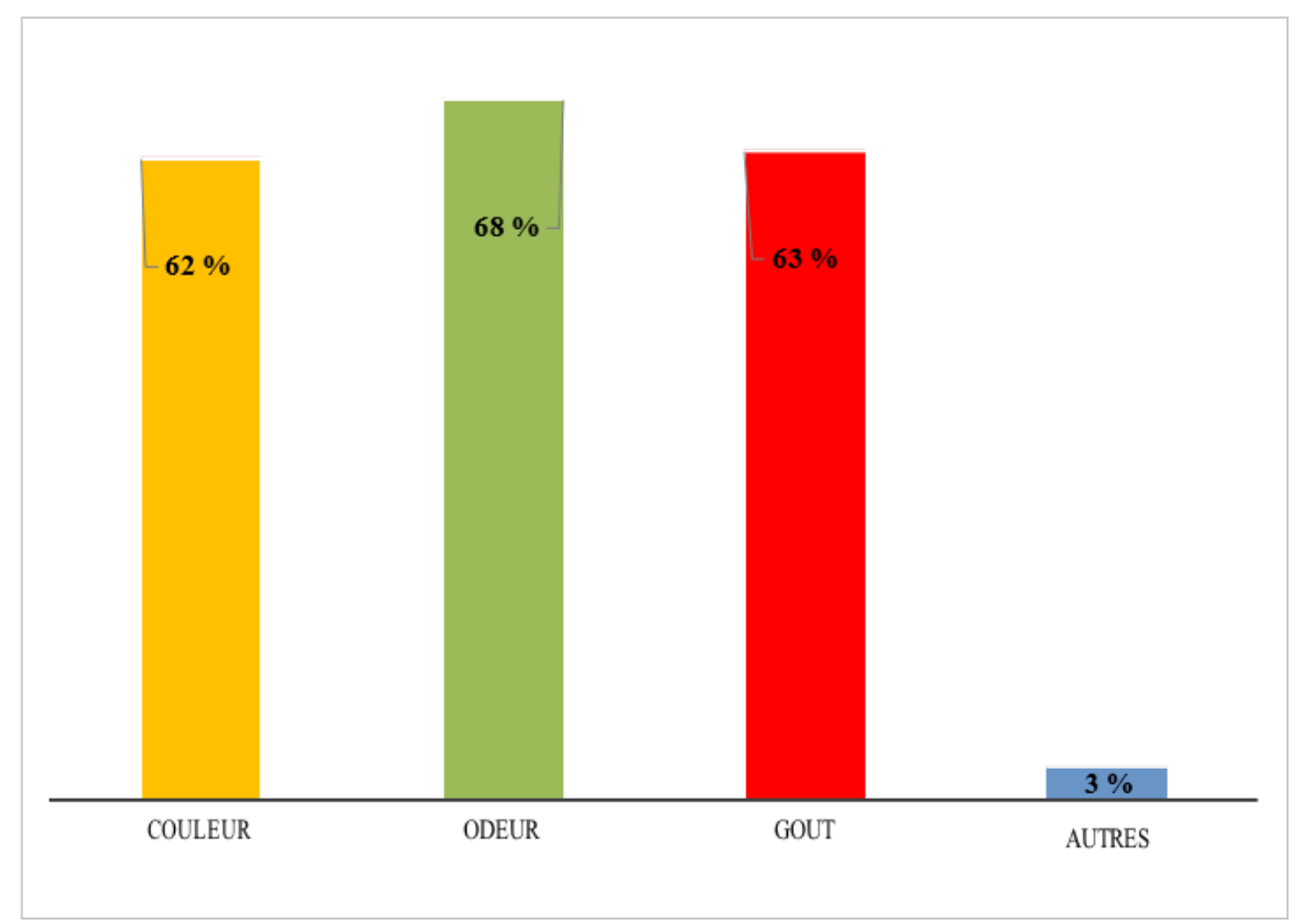

Figure 6 : Critères d'appréciation de la qualité de l'eau par la population étudiée

L'odeur est le paramètre organoleptique le plus utilisé par la population (68 $\%)$ pour apprécier la qualité de l'eau suivi du goût (63\%) et de la couleur (62 $\%)$. Selon certaines études, ce résultat peut s'expliquer par le caractère corrosif et incrustant de l'eau qui est une cause de l'altération du goût et de l'aspect dans le réseau d'adduction (Doria, 2007 ; Fabbricino et Korshin, 2014 ; Gunnarsdottir et al., 2016). En effet, ce sont les qualités esthétiques de l'eau, plus spécialement celles reliées à la couleur, aux goûts et aux odeurs, qui peuvent constituer une part importante de la perception du consommateur relative à l'eau potable (OMS, 2017). Peu de gens parmi la population prennent en considération d'autres paramètres que ceux organoleptiques pour apprécier la qualité de l'eau alors que selon (Ochoo et al., 2017), la perception de la qualité de l'eau par le consommateur est souvent différente de la qualité sanitaire de l'eau mesurée. Cette situation pourrait être à l'origine de l'utilisation d'autres sources d'eau de meilleure apparence mais dont la salubrité n'est pas garantie.

\subsection{Moyens de traitement de l'eau}

Un mauvais goût, une présence de coloration et une mauvaise odeur sont souvent les raisons pour lesquelles l'eau du robinet et des pompes sont traitées à domicile avant leur usage. Il est à noter que l'eau du robinet privé est celle qui a le plus fait l'objet de traitement que les autres types d'eau. Les 
différents moyens de traitement utilisés par les populations pour rendre l'eau salubre sont présentés à la figure 7 .

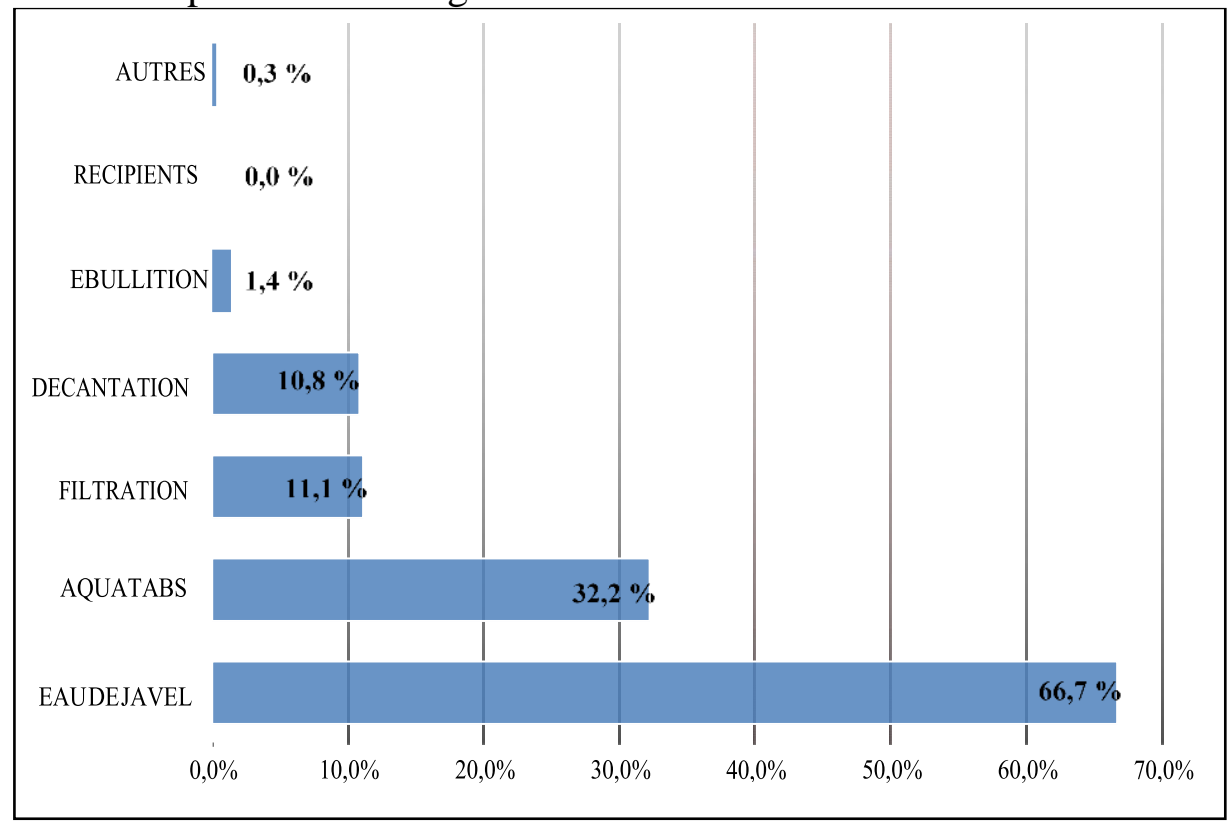

Figure 7 : Moyens de traitement de l'eau dans la commune des Parcelles Assainies

L'eau de javel est le moyen de traitement de l'eau le plus utilisé $(66,7$ $\%)$, suivi d'Aquatabs ${ }^{\circledR}(32,2 \%)$. La filtration et la décantation sont aussi à noter avec respectivement $11,1 \%$ et $10,8 \%$ de procédés de traitement de l'eau. L'ébullition est très peu appliquée et la méthode des trois récipients n'est pas utilisée. Ces résultats sont en opposition avec ceux observés en milieu rural où (Diop et al., 2019) constatent que les techniques de traitement utilisées sont essentiellement la filtration (30\%), la décantation (17\%) et la désinfection à l'eau de Javel (15\%). De même, (Biembe, 2019) montre que dans certains quartiers de Yaoundé au Cameroun, $63 \%$ des ménages traitent l'eau avec des filtres multi-étages, $13 \%$ pratiquent la chloration, $8 \%$ la décantation.

\subsection{Qualité de l'eau et santé des enfants}

Les méthodes de traitement de l'eau à domicile ne peuvent améliorer la qualité de l'eau et prévenir les maladies que si elles sont utilisées correctement. Ainsi, la plupart des individus qui vivent dans la commune des Parcelles Assainies (75\%) pense qu'il existe un rapport entre la qualité de l'eau et la santé des enfants âgés de moins de 5 ans (Figure 8). 


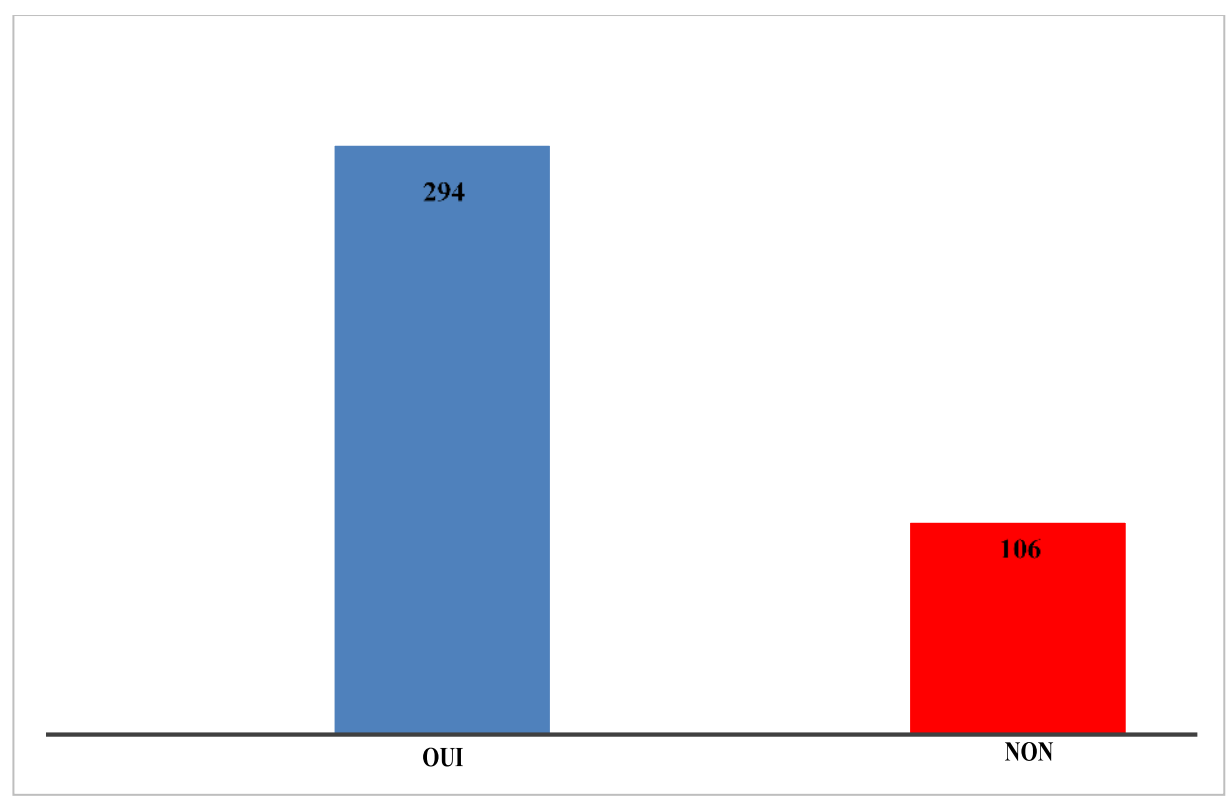

Figure 8 : Perception du rapport entre la qualité de l'eau et les maladies diarrhéiques

Selon une étude de l'UNICEF, le manque d'eau salubre et de moyens d'assainissement est la première cause de maladies dans le monde particulièrement chez les enfants dont 4500 en meurent chaque jour (UNICEF, 2007). En outre, dans un souci d'hygiène, l'eau ne doit pas être utilisée seule lors du nettoyage des mains. A cet effet, elle est accompagnée de produits détergents, antiseptiques et savonneux. Toutefois, une partie des habitants $(14,3 \%)$ se limite à l'eau seulement sans détergent pour le lavage des mains à la sortie des toilettes. Ce qui est en opposition avec les recommandations de l'OMS qui sont de se laver les mains à l'eau et au savon lorsqu'elles sont visiblement souillées par du sang ou d'autres liquides biologiques ou après être allé aux toilettes (OMS, 2009). Cet état de fait peut, en plus de l'insalubrité de l'eau de boisson, être à l'origine des maladies diarrhéiques chez les enfants. En effet, quatre millions de décès d'enfants par an sont imputables à l'eau insalubre, principalement, de par la diarrhée qu'elle occasionne chez ce groupe d'âge et les pays en développement sont les plus concernés par cette forte charge de mortalité (Kaboré et al., 2017). Nos résultats sont par contre en contradiction avec ceux de (Ballet et al., 2018) qui révèlent que 85,3\% et 79,4 $\%$ des ménages enquêtés respectivement dans la commune de Yopougon et Cocody n'ont aucune connaissance quant aux risques sanitaires liés à la consommation de l'eau du robinet, $12 \%$ et $17 \%$ pensent que l'eau du robinet ne présentait aucun risque, contre $3 \%$ et $4 \%$ qui pensent qu'elle peut présenter des risques mineurs respectivement. 


\section{Conclusion}

$\mathrm{Au}$ Sénégal, l'approvisionnement en eau potable constitue un problème majeur de santé publique. Malgré les politiques mises en œuvre pour améliorer l'approvisionnement en eau potable, l'accessibilité à cette denrée est limitée et conduit la population à l'utilisation d'autres sources d'eau de qualité douteuse. Cette situation soulève des inquiétudes relatives à la qualité et à l'impact de ces eaux sur la santé de la population, particulièrement des enfants âgés de moins de 5 ans. Même si l'eau du robinet est à la portée de la presque totalité de la population, cette dernière n'est pas satisfaite de sa disponibilité et de sa salubrité de par son aspect, son goût et son odeur. De ces faits, des moyens de potabilisation des eaux du robinet et des pompes comme l'eau de javel en général ou l'Aquatabs® en particulier sont utilisés à domicile avant toute consommation. Toutefois, la non maitrise des techniques de traitement associée à des conditions d'hygiène et d'assainissement précaires constituent un danger pour la santé surtout des enfants qui sont les plus sensibles aux germes pouvant contaminer l'eau et les aliments. En ce sens, la majorité des individus vivant dans la commune considère que le mauvais état de santé des enfants âgés de moins de 5 ans est lié à la qualité de l'eau. Ce travail a été mené afin de montrer au décideur qu'assurer une disponibilité continue de l'eau potable à toute la population réduirait la mortalité et la morbidité des maladies transmises par l'eau.

\section{References :}

1. ANSD Agence Nationale de la Statistique et de la Démographie (2016). La population du Sénégal en 2016 : Un extrait des projections démographiques du RGPHAE 2013, Dakar, Sénégal, 33 p.

2. ANSD Agence Nationale de la Statistique et de la Démographie (2018). Rapport de l'Enquête Démographique et Sanitaire continue au Sénégal. $5^{\text {ème }}$ édition, Dakar, Sénégal, 644 p.

3. Ballet, T.G.N., Gnagne, A.E.J.E.Y., Fofana, V. \& Yapo B.O. (2018). Évaluation de la perception des ménages de la qualité de l'eau du robinet de deux communes, Cocody et Yopougon de la ville d'Abidjan, Côte d'Ivoire. Afrique SCIENCE, 14(6), 48-57.

4. Barou, J. (2017). Dynamiques de transformation familiale en Afrique subsaharienne et au sein des diasporas présentes en France. Droit et cultures. Revue Internationale Interdisciplinaire, (73), 29-47.

5. Biembe, Y.J. (2019). Risques sanitaires liés à la potabilisation domestique de l'eau dans les quartiers à habitats spontanés de Yaoundé : cas du bassin versant de l'Abiergue, Mémoire de Master, 82 p, Université de Liège, Belgique.

6. Brooks, D.B. (2002). L'eau : Gérer localement. CRDI, Ottawa, Canada, 92p. 
7. Caritas Sénégal (2009). Impact du fluor ingéré via les eaux de boisson sur la santé des populations de 5 communautés rurales des départements de Fatick, Mbour ; Rapport 1/2, 43 p, Dakar, Sénégal.

8. Diop, C., Toure, A., Cabral, M., Thiandoum, M. \& Fall, M. (2019). Évaluation des pratiques de potabilisation des eaux de boisson en zone rurale : cas de Sindia au Sénégal. Afrique SCIENCE 15(3), 253-260.

9. Doria, M.F. (2010). Factors influencing public perception of drinking water quality. Water Policy, 12, 1-19.

10. Dupont, C., Campagne, A. \& Constant, F. (2014). Efficacy and safety of a magnesium sulfate-rich natural mineral water for patients with functional constipation. Clinical Gastroenterology and Hepatology, 12(8), 1280-1287.

11. Fabbricino, M. \& Korshin, G. V. (2014). Changes of the corrosion potential of iron in stagnation and flow conditions and their relationship with metal release. Water Research, 62(1), 136-146.

12. Gunnarsdottir, M.J., Gardarsson, S.M., Jonsson, G.S. \& Bartram, J. (2016). Chemical quality and regulatory compliance of drinking water in Iceland. Int. J. Hyg. Environ. Health, 219(8), 724-733.

13. Hanus, J. (2005). Evaluation de la Salubrité de l'eau des puits de Pointe-Noire. Caractérisation et Etudes de Santé. Université Catholique de Louvain, DEA en science et l'environnement : $3^{\text {ème }}$ Partie : Aspects de Santé Publique. Louvain, 87 p.

14. He, Z., Bishwajit, G., Zou, D., Sanni, Y., Zhaohui, C. \& Yan, Z. (2018). Burden of Common Childhood Diseases in Relation to Improved Water, Sanitation, and Hygiene (WASH) among Nigerian Children. Int. J. Environ. Res. Public Health, 15, 1241.

15. Kaboré, A., Savadogo, B., Sawadogo, J., Konate, A., Savadogo, P.W. Kabore, I.B., Nikiema, Z.P.L. \& Dianou, D. (2017). Problématique de la qualité microbiologique de l'eau de boisson en milieu scolaire dans les zones rurales au Burkina Faso. Rev. Cames, 05, (02) : 13-17.

16. Makoutode, M., Assani, A.K., Ouendo, E.M., Agueh, V. D. \& Diallo P. (1999). Qualité et mode de gestion de l'eau de puits en milieu rural au Benin : cas de la sous-préfecture de Grand-Popo. Médecine d'Afrique Noire, 46(11), 474-478.

17. Nanfack, N.A.C., Fonteh F.A., Payne, V.K., Katte, B. \& Fogoh J.M. (2014). Eaux non conventionnelles: un risque ou une solution aux problèmes d'eau pour les classes pauvres. Larhyss Journal, 17, 47-64.

18. Nkounkou, C.L., Louzayadio, M.R.F., Ayessou, N., Elouma, N.A.M., Ngakegni-Limbili, A.C., Mar-DIOP, C.G. \& Ouamba, J.M. (2017). Approvisionnement en eau dans la ville de Brazzaville, Congo. European Scientific Journal, 13(21), 474-498. 
19. Ochoo, B., Valcour, J. \& Sarkar, A. (2017). Association between perceptions of public drinking water quality and actual drinking water quality: A community-based exploratory study in Newfoundland (Canada). Environ Res., 159, 435-443.

20. OMS (2007). Atteindre l'OMD relatif à l'eau potable et à l'assainissement le défi urbain et rural de la décennie. OMS, Genève, Rapport sur l'eau et l'assainissement, $48 \mathrm{p}$

21. OMS (2009). Hygiène des mains: pourquoi, comment et quand. OMS, Genève, Rapport sur le lavage des mains, $7 \mathrm{p}$.

22. OMS (2017). Directives de Qualité de l'eau de boisson. OMS, Genève, $4^{\text {ème }}$ Edition, $564 \mathrm{p}$.

23. Popoff, G. (2010). Spécificité, législation et contrôle des eaux minérales naturelles utilisées dans les établissements thermaux français. Presse Thermale et Climatique, 147, 93-119.

24. Proulx, F., Rodriguez, M. \& Sérodes, J.B. (2010). Tastes and odours in drinking water: a review of related compound and measurement techniques, Journal of Water Science, 23(3), 197-323.

25. Roch, L. (2008). Au croisement entre pénurie d'eau et société du risque: un nouvel éclairage sur la problématique de l'eau. Mémoire de Maitrise, Université du Québec, Montréal, 146 p.

26. Santsa-Nguefack C.V., Ndjouenkeu, R. \& Ngassoum, M.B. (2018) Qualité de l'eau de la localité de Dschang et impact sur la santé des consommateurs. Afrique SCIENCE, 14(3), 96-107.

27. Sène, E. (2012). Approvisionnement en eau potable dans la commune de Fatick. Mémoire de Master, Université Cheikh Anta Diop, Dakar, $77 \mathrm{p}$.

28. Tchouongsi, E.K., Mougoue, B., Tagne, C.T., Touoyem, F.M. \& Bonganjum, N.S. (2020). Approvisionnement en eau et risques sanitaires dans le bassin versant amont de l'Abiergué à Yaoundé (Cameroun). European Scientific Journal, 16(8), 102-123.

29. Thiam, S., Sy, I., Schindler, C., Niang-Diène, A., Faye, O., Utzinger, J. \& Cisse, G. (2019). Knowledge and pratices of mother and caragivers on diarrhoeal management among under 5-year-old children in a medium-size town of Senegal. Acta tropica, 194, 155164.

30. UNICEF (2007). Atteindre l'Objectif du Millénaire pour le Développement relatif à l'eau potable et à l'assainissement : le défi urbain et rural de la décennie. UN Plaza, New York, NY 10017, EtatsUnis d'Amérique, $48 \mathrm{p}$.

31. UNICEF et OMS (2019). Progress on household drinking water, sanitation and hygiene $2000-2017$ : special focus on inequalities. 3 UN Plaza, New York, NY 10017, Etats-Unis d'Amérique, 138 p. 